\title{
Structure of the Milky Way and the distribution of young stellar clusters
}

\author{
Maria Messineo ${ }^{1}$, Karl M. Menten ${ }^{2}$, Harm J. Habing ${ }^{3}$, \\ Monika Petr-Gotzens ${ }^{1}$ and Frédéric Schuller ${ }^{2}$ \\ ${ }^{1}$ ESO, Garching, Germany \\ email: mmessine@eso.org \\ ${ }^{2}$ MPIfR, Bonn, Germany \\ ${ }^{3}$ Leiden Observatory, The Netherlands
}

\begin{abstract}
We present infrared observations with the integral field spectrometer SINFONI on the ESO-VLT (Very Large Telescope) Yepun of obscured stellar clusters in HII regions. The classification in spectral subclasses of the detected early type stars together with a determination of the interstellar extinction and the stellar apparent magnitudes enable us to obtain spectrophotometric distances, which are independent and complementary to kinematic distances and free from the ambiguity inherent to the determination of the latter. This study is part of a larger project, investigating the spatial distribution of Galactic HiI regions. Our primary aim is obtaining a more complete and less biased picture of the Milky Way's spiral structure.
\end{abstract}

Keywords. (Galaxy:) open clusters and associations: general Galaxy: stellar content

\section{Introduction}

Galactic clusters are continuously formed in the disk of a spiral galaxy, typically along the spiral arms. During the earliest stages of evolution the clusters are completely embedded in their parental molecular clouds. The identification of embedded stellar clusters in the Galactic plane and the study of their spatial distribution are therefore of primary importance for a better understanding of the current star formation rate and of the Galactic structure.

Embedded clusters are good tracers of the spiral arms, but their distance determinations often rely only on radial velocities from radio data. There is an intrinsic ambiguity when using kinematic distances inside the solar circle: each radial velocity measurement is consistent with a near and a far solution for the distance. Furthermore, it depends on the assumed model of Galactic rotation. Nowadays the actual number and location of spiral arms is still uncertain Russeil (2003). Furthermore, only a few young and massive clusters (the Quintuplet, Arches, and Sgr A clusters) are known to reside inside the central $3 \mathrm{kpc}$, all of them concentrated within the central 50 parsecs.

The advent of new and powerful infrared detectors opened a new era of Galactic structure studies. It is now possible to observe highly extincted HiI regions at large distances from the Sun. The recent availability of large mid- and near-infrared surveys of the Galactic plane, such as 2MASS, ISOGAL, MSX and GLIMPSE has led to the discovery of several hundreds of new Galactic HiI regions, and candidate infrared stellar clusters. We have compiled a list of 647 Galactic young clusters which were mostly selected by inspecting 2MASS images at the position of known HiI regions, e.g by Bica, et al. (2003). Their young nature is confirmed by associated continuum emission at 1.4 $\mathrm{GHz}$ (NRAO VLA Sky Survey) - for $85 \%$ of them. We expect to find many new candidate inner Galactic clusters with the ongoing UKIDSS survey, a deeper near-infrared survey of the Galactic plane, and with future VISTA surveys. 
Infrared follow-up studies are required in order to resolve each cluster into individual stars and to obtain stellar spectra that provide a complete census of the brightest stars and, thus, allow the determination of the spectro-photometric distance to the cluster.

Aiming to improve our understanding of the spatial distribution of Galactic HiI regions we have started a program with the integral field spectrometer SINFONI on the ESOVLT (Very Large Telescope) Yepun to observe obscured stellar clusters in HiI regions. We have obtained observations of eight obscured stellar clusters in Hir regions - in W31, W42, W43, W51 and the cluster [DBS2003]8 - in order to determine their spectrophotometric distance. The program includes observations of candidate OB stars in both the $\mathrm{H}(1.45$ $-1.85 \mathrm{~mm})$ and $\mathrm{K}(1.95-2.45)$ bands at the highest SINFONI resolution ( $\mathrm{R} 3000$ and 4000, respectively).

By performing integral field spectroscopy with SINFONI we simultaneously image and resolve the core of the stellar clusters and obtain spectra (e.g. Messineo, et al. (2006)). Since often in one field more than one star can be measured, it is typically possible to obtain a complete census of the cluster's brightest stars by employing a few telescope offsets in a single $1 \mathrm{hr}$ observation sequence. Furthermore, SINFONI enables us to detect simultaneously nebular emission (such as molecular hydrogen lines, HI recombination lines and ionized iron) and the spectrum of the candidate ionizing star, allowing us to distinguish between these contributions. We have successfully detected photospheric lines in several stars of each cluster considered.

Through the detection and spectral classification of individual cluster OB stars we are able to obtain a spectrophotometric distance to the cluster. Such distance estimates are independent and complementary to kinematic distances and will provide new constraints on the Galactic morphology. Most importantly, our method is based on near-infrared observations, which are much less limited by interstellar extinction than previous optical programmes, allowing studies along lines of sight where optical measurements are impossible.

\section{2. $[$ DBS2003] 8}

We performed pilot observations of [DBS2003]8, an embedded Galactic stellar cluster in the bright rimmed cloud SFO49, during the SINFONI science verification Messineo, et al. (2006).

The $\mathrm{K}$-band brightest star, which is located in the cluster center, presents spectral lines typical of a late $\mathrm{O}$ or early $\mathrm{B}$ type dwarf star and is surrounded by an, almost entirely spherical, ionized nebula. We derive a spectrophotometric distance to the cluster of $2.5 \pm 0.7 \mathrm{kpc}$ by assuming the central source being a zero-age main-sequence star with spectral type between O9 and B1. The nebula has an observed radius of $\sim 0.03-$ $0.06 \mathrm{pc}$, which is a typical value for an ultra-compact HiI region. We measured the nebula's Br $\gamma$ emission, generated by photo-ionization of the central star and from the hydrogen molecular lines ratio we proved that the ionized nebula is surrounded by a photo dissociated region (PDR).

\section{References}

Bica, E., Dutra, C. M. \& Barbuy, B. 2003 A $3 A 397177$

Messineo, M., Petr-Gotzens, M. G., Schuller, F., Menten, K.M., Habing, H.J., Kissler-Patig, M., Modigliani, A., \& Reunanen, J. 2006, submitted to $A \mathscr{E} A$

Russeil, D. 2003 AESA 397133 